\title{
DIRECT ADAPTIVE REJECTION OF VORTEX-INDUCED DISTURBANCES FOR A POWERED SPAR PLATFORM
}

\author{
Tannen S. VanZwieten \\ Aerospace Engineer \\ NASA Marshall Space Flight Center \\ Huntsville, AL 35812 \\ Email: tannen.s.vanzwieten@nasa.gov
}

\author{
Mark J. Balas \\ Department Head and Professor \\ Dept. of Electrical \& Computer Engineering \\ University of Wyoming \\ Laramie, Wyoming 82071 \\ Email:mbalas@uwyo.edu
}

\author{
James H. VanZwieten* \\ Research Professor \\ Dept. of Ocean Engineering \\ Florida Atlantic University \\ Dania Beach, Florida 33004 \\ Email: jvanzwi@fau.edu
}

\author{
Frederick R. Driscoll \\ Associate Professor \\ Dept. of Ocean Engineering \\ Florida Atlantic University \\ Dania Beach, Florida 33004 \\ Email: rdriscol@fau.edu
}

\section{ABSTRACT}

The Rapidly Deployable Stable Platform (RDSP) is a novel vessel designed to be a reconfigurable, stable at-sea platform. It consists of a detachable catamaran and spar, performing missions with the spar extending vertically below the catamaran and hoisting it completely out of the water. Multiple thrusters located along the spar allow it to be actively controlled in this configuration. A controller is presented in this work that uses an adaptive feedback algorithm in conjunction with Direct Adaptive Disturbance Rejection (DADR) to mitigate persistent, vortex-induced disturbances. Given the frequency of a disturbance, the nominal DADR scheme adaptively compensates for its unknown amplitude and phase. This algorithm is extended to adapt to a disturbance frequency that is only coarsely known by including a Phase Locked Loop (PLL). The PLL improves the frequency estimate on-line, allowing the modified controller to reduce vortexinduced motions by more than $95 \%$ using achievable thrust inputs.

\section{LIST OF ACRONYMS}

DADR

DGPS

DOF

FAU

HTM

LF

LTI

MIMO

MRAC

PID

PLL

RDSP

SISO

STD

VIV

VOM
Direct Adaptive Disturbance Rejection Differential Global Positioning System Degrees Of Freedom

Florida Atlantic University

Horizontal Transit Mode

Loop Filter

Linear Time-Invariant

Multi Input Multi Output

Model Reference Adaptive Control

Proportional Integral Derivative

Phase Locked Loop

Rapidly Deployable Stable Platform

Single Input Single Output

STandard Deviation

Vortex Induced Vibration

Vertical Operating Mode

${ }^{*}$ Address all correspondence to this author. 


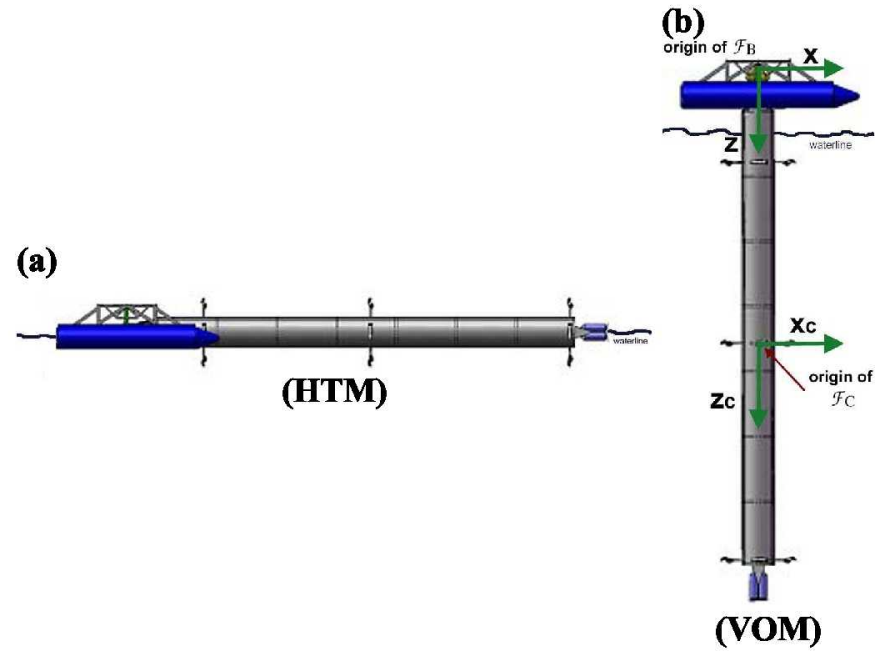

Figure 1. THE RDSP'S TWO OPERATIONAL MODES.

\section{INTRODUCTION}

Quasi-steady currents around a spar can result in disturbance-generating vortices. These are shed at relatively low frequencies [1], and the resulting forces are nearly periodic and somewhat theoretically predictable [2]. These frequencies can be estimated empirically, but cannot be precisely determined using existing analytic means. Their effect has been studied for offshore structures in [3], and specifically for cylinders or spar platforms in $[2,4,5]$. If the frequency of vortex shedding is in resonance with the natural frequency of the moored structure that produces it, large amplitude Vortex-Induced Vibrations (VIVs) can develop, introducing large stresses on the member and mooring lines [1]. The use of active control for moored spar platforms to mitigate VIVs was introduced in [2]. For a spar with a length of $198 \mathrm{~m}$ and a diameter of $38 \mathrm{~m}$, sixteen $3000 \mathrm{hp}$ thrusters were recommended at a total cost of between 7 and 8 million dollars [2]. This proposed active control system resulted in a significant reduction in installation cost when compared to the typical passive VIV-control devices (such as helical strakes).

This paper will consider vortex-induced disturbances on an un-moored powered spar platform called a Rapidly Deployable Stable Platform (RDSP). The RDSP is currently under development to create a mobile yet stable offshore platform for multiple applications. It consists of two elements: $i$ ) a catamaran and $i$ ) a long, detachable spar buoy, as depicted in Fig. 1. The RDSP can be configured for two modes of operation: Horizontal Transit Mode (HTM) and Vertical Operations Mode (VOM). The majority of the potential missions for the RDSP will be conducted when it is operating in VOM, where spar extends vertically below the catamaran and lifts it completely out of the water (Fig. 1). This spar configuration presents a small cross-sectional area to passing waves, thereby improving its seakeeping properties. The spar component of the RDSP is equipped with 12 thrusters for control in the VOM. These thrusters allow the RDSP to transit at slow speeds, dynamically position, and mitigate disturbances. A one tenth scale model of a RDSP (11.4 m spar length) has been designed and constructed at Florida Atlantic University (FAU), complete with a sensor suite that is capable of providing all of the states of the RDSP [6]. Preliminary findings for a full scale RDSP with a $10 \mathrm{~m}$ diameter reveal vortex-induced oscillations with displacement amplitudes of approximately $1 \mathrm{~m}$ [7]. This is significant when dynamically positioning near other vessels for cargo transfer applications. Mitigating these disturbances for unmoored applications is the focus of this paper.

An adaptive control approach that uses Direct Adaptive Disturbance Rejection (DADR) to mitigate vortex-induced disturbances will be taken in this paper. The disturbance rejection algorithm will be appended with a Phase Locked Loop (PLL) for on-line disturbance frequency estimation. This is suitable for mitigating vortex-induced RDSP motions since they are associated with eddy shedding frequencies that can be estimated but cannot be precisely determined. The controller will take advantage of the known and estimated disturbance properties (waveform and initial frequency guess) and adaptively compensate for the unknown or estimated components (amplitude, phase and frequency). Results will be simulated using a 6 Degree Of Freedom (DOF) computer simulation of this powered ocean platform developed in $[8,9]$.

The development of the adaptive control and disturbance rejection algorithm used here may be found in [10], prior to augmenting it with a PLL. This was previously applied to the RDSP without the inclusion of frequency synthesis for wave and load disturbance rejection in [11-13]. The concept of augmenting the algorithm with a PLL in order to reject disturbances at coarsely known frequencies was considered in [7,14], and applied to a simplified 1-DOF RDSP simulation. This paper extends the work of [7] by providing a more detailed description and analysis of the PLL, and applying it to a more realistic and complex simulation of the RDSP that utilizes all 6-DOF (rather than 1-DOF). Furthermore, multiple sensor and actuator configurations will be considered.

The paper will be organized in the following manner. First, we will begin with a summary of the 6 DOF model for the RDSP, including the current-driven vortex forces. Next, the adaptive control and disturbance rejection framework will be introduced, including a modification for disturbances at frequencies that are not precisely known. Simulation results are shown for two RDSP scenarios: (i) a single output at the top of the spar controlled using a single force located at the center thruster (ii) control actuation via force and moment generated at the center thruster with two collocated outputs. Finally, some concluding remarks will be made. 


\section{NUMERICAL MODEL OF THE $1 / 10^{T H}$ SCALE RDSP PROTOTYPE}

The control systems presented in this paper are developed and tuned to control the $1 / 10^{\text {th }}$ scale RDSP prototype designed and constructed at Florida Atlantic University. The initial design of this system is presented by [15] and the upgraded prototype is presented in [8] and briefly reviewed here. This prototype has a cylindrical spar section with a length of $11.4 \mathrm{~m}$ and a diameter of $0.84 \mathrm{~m}$ (Fig. 1). The spar is attached via a hinge to a catamaran with pontoons that both have lengths of $4.1 \mathrm{~m}$ and diameters of $0.42 \mathrm{~m}$. The catamaran has a dry mass of $361 \mathrm{~kg}$ while the spar has a total dry mass of $1,437 \mathrm{~kg}$, can hold $1,317 \mathrm{~kg}$ of ballast water, and has $4,791 \mathrm{~kg}$ of entrained sea water, not including ballast water, when fully submerged. When operating in VOM (Fig. 1 (b)) the catamaran and spar are rigidly locked together forming a single nearly rigid body that has a total wet mass including entrained sea water of up to $7,906 \mathrm{~kg}$. When configured in HTM (Fig. 1 (a)), the ballast tanks are only partially filled with water and the entrained sea water is reduced, thereby reducing the total wet mass of the RDSP.

The 1/10th scale RDSP prototype has 12 thrusters that are located on the spar to allow the RDSP to actively control its position and orientation (Fig. 1). These thrusters are automatically controlled using a computer system that links state feedback from a comprehensive sensor suite including an IMU, dual antenna DGPS compass, and tilt sensor to thruster inputs. Each of these thrusters can individually produce a maximum forward thrust of $320 \mathrm{~N}$ and a maximum reverse thrust of $170 \mathrm{~N}$. This is much greater than the maximum scaled thrust of $47 \mathrm{~N}$ per thruster estimated from the proposed generator system suggested for the full scale system $[8,9]$. Not only is this system controllable using thrusters, but it also has an active ballasting system that can be used to change the spar's inertial properties, raise and lower the catamaran, and transfer the RDSP prototype between HTM and VOM.

\section{6-DOF Mathematical Model}

A 6-DOF mathematical model and computer simulation of the $1 / 10^{\text {th }}$ scale RDSP prototype has been developed. This simulation models drag, added mass, wind, vortex, and hydrostatic forces on the RDSP.

Kinematics When the catamaran and spar are rigidly connected in VOM (Fig. 1 (b)), the RDSP can be modeled as a rigid body with its states defined using two coordinate systems. These coordinate systems are the earth fixed coordinate system, $\mathcal{F}_{\mathrm{E}}$, denoted by capital variables and body fixed coordinate system, $\mathcal{F}_{\mathrm{B}}$, denoted by lowercase variables. The earth fixed coordinate system, $\mathcal{F}_{\mathrm{E}}$, has its origin at the surface of the mean seawater with the $X$-axis pointing North, $Y$-axis pointing East, and $Z$-axis pointing down. The body fixed coordinate system, $\mathcal{F}_{\mathrm{B}}$, is fixed to the RDSP with its origin where the spar and the catamaran attach and the $x$-axis pointing towards the bow of the catamaran, the $y$ axis pointing towards starboard, and $z$-axis pointing downwards along the spar; in accordance with the right hand rule (Fig. 1 (b)). An additional coordinate system is used by the MIMO controller, $\mathcal{F}_{\mathrm{C}}$, has its origin midway between the center four thrusters with its axes aligned with those of $\mathcal{F}_{\mathrm{B}}$ and defined by $x_{\mathrm{C}}, y_{\mathrm{C}}$, and $z_{\mathrm{C}}$ (Fig. 1 (b)).

Drag and Added Mass Hydrodynamic drag and added mass forces are modeled using Morison's equation, which accounts for the interactions between wave, current, and body (catamaran and spar) motions [16],

$$
f=\frac{1}{2} C_{\mathrm{d}} \rho A \bar{u}|\bar{u}|+C_{a} \rho \dot{\bar{u}} V,
$$

where $\rho$ is the density of sea water, $(\overline{)})$ indicates that the relative velocity or acceleration of the water or air with respect to the component that is being referenced, $A$ is the vector of projected areas in the $x$ - and $z$-directions, $V$ is the submerged volume, $C_{\mathrm{d}}(R e)$ is the vector of coefficients of drag in the $x$-, $y$-, and $z$-directions, $C_{a}$ is the vector of coefficients of added mass in the $x$-, $y$-, and $z$-directions, and $f$ is the combined drag and added mass force vector in the $x-, y$-, and $z$-directions. The drag force portion of this equation is a combination of skin friction and residual drag.

For the spar, skin drag is modeled only for a longitudinal flow along its length (parallel to the $z$-axis) and for the catamaran, skin drag is modeled only for a longitudinal flow along their lengths (parallel to the $x$-axis). The skin drag coefficient used for the spar and catamaran is calculated using the Reynolds number calculated along the length of the spar and pontoons respectively. The skin drag coefficients used for the longitudinal flows, $C_{\mathrm{d}}^{\text {skin }}$, are calculated as suggested by [17],

$$
C_{\mathrm{d}}^{\mathrm{skin}}=\frac{0.0075}{\left(\log _{10}(R e)-2\right)^{2}}
$$

The skin drag force varies nonlinearly along the length of the spar as the relative water velocity varies along the spar and catamaran pontoons lengths and drag force is a nonlinear function of this relative velocity. For this reason, numerical integration is used to calculate the net skin drag force along the spar and catamaran pontoons.

Residual drag is calculated on the spar body, spar thrusters, and catamaran pontoons. For the spar, residual drag is calculated for a flow across its body, on the bottom end (bow), and on its thrusters. For the catamaran, residual drag is calculated for a flow perpendicular to the pontoons and along the pontoons at 
their ends. Strip theory is used to calculate the forces perpendicular to the spar and pontoon buoys while a constant relative water velocity is assumed for the ends of the spar and catamaran pontoons. The residual drag coefficient for a flow across the spar ( $x$-direction) is assumed to have a constant value of 1.0, as suggested by [18]. This same coefficient is used to calculate the residual drag across the pontoons (z-direction) as both the pontoons and the spar are approximately cylindrical. The coefficient or residual drag for a flow along the spar (z-direction) at the submerged end is also assumed to have a value of 1.0 , as suggested by [18]. The same coefficient is used for the residual drag on the ends of the pontoons. Residual drag forces on the submerged thrusters are calculated independent from the rest of the spar as they are the only components of the RDSP that protrude out from the spar's frame. The residual drag force on a thruster is calculated using separate but constant coefficients for flow parallel and perpendicular to the thrust axis. These coefficients are derived from tow tank tests, where the thrusters are towed facing parallel and perpendicular to the tow direction. The total drag coefficients are derived from fitting curves to the data, which are a function of velocity squared. These coefficients are used to calculate the drag forces for a flow parallel to each thruster and for a flow perpendicular to each thruster.

Added mass forces for both the spar and catamaran are modeled as an increase to the virtual mass and moment of inertia of the RDSP. This simplification can be made since waves are not considered for this application and therefore the local RDSP acceleration is the local relative acceleration. When operating in VOM, the virtual mass and virtual moment of inertia are calculated for the RDSP in each direction using the actual mass and moment of inertia (including entrained water), the added mass and moment of inertia from the spar, and the added mass and moment of inertia from the catamaran. The coefficient of added mass for spar acceleration in the $x$-direction, perpendicular to the spar, is chosen as 1 and the coefficient of added mass for spar acceleration in the $z$-direction, parallel to the spar, is chosen as $1 / \pi$ as suggested by [18]. These same coefficients are used for a flow perpendicular and parallel to the catamaran pontoons, respectively.

Thruster Forces The individual thruster forces and thrust rate are limited to match the maximum thrust to the power plant suggested by [19] using Froude scaling. This limits the maximum thrust per engine to $47 \mathrm{~N}$ and the maximum thrust rate of change per engine to $37.2 \mathrm{~N} / \mathrm{s}$ for the motor configuration chosen for the $1 / 10^{\text {th }}$ scale RDSP prototype with thrust values scaled from Thrustmaster Model TH 300N azimuthing thruster [20].

\section{MODEL OF VORTEX-INDUCED DISTURBANCES ON THE RDSP}

Vortex-induced forces on spar platforms, such as the RDSP, develop when eddies are shed behind the cylindrical spar element as it moves relative to the surrounding fluid (Fig. 2). This occurs

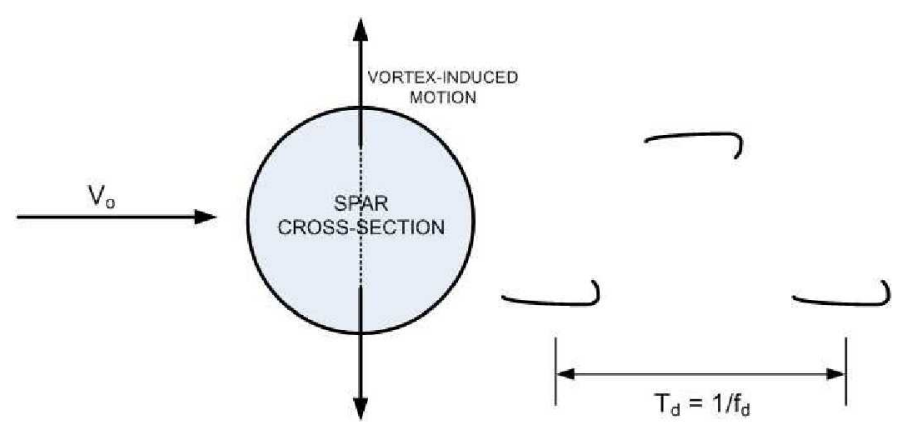

Figure 2. VORTEX-INDUCED MOTION ON A CIRCULAR CROSSSECTION.

when the relative velocity vector of the fluid has a component that acts parallel to the cross-section of the spar. During most operations, the RDSP will be dynamically positioned or moored in a quasi-steady current. This induces disturbance-generating vortices around the spar, beginning at very low relative water velocities. Vortices will also be shed at a nearly steady frequency and amplitude when the RDSP is traveling in VOM at a nearly constant speed relative to the water velocity, such as when transferring cargo from a slowly moving vessel. The frequency of these eddies can be estimated, but precise knowledge of the vortex shedding frequency cannot be determined analytically.

\section{Eddy Shedding Frequency}

For a spar with a given diameter, the eddy shedding frequency can be approximated using the relative water velocity [5]. The frequency of shedding vortices from one side (see Fig. 2), $\omega_{\mathrm{d}}=2 \pi f_{\mathrm{d}}=\frac{2 \pi}{T_{\mathrm{d}}}$, is also the frequency of the oscillatory motion induced in the transverse direction. This is typically estimated empirically as a function of the Strouhal number with a relationship defined by [1] as

$$
\omega_{\mathrm{d}}=2 \pi \frac{S V_{0}}{D}
$$

where $S$ is the Strouhal number, $D$ is the diameter, and $V_{0}$ is the relative free stream velocity. The Strouhal number itself varies according to a poorly defined, nonlinear function of the current velocity. A relationship given by [1] shows that the Strouhal number ranges from 0.18 to 0.32 . However, it can be approximated as a constant with a value of 0.21 for cylindrical bodies, 
as suggested by [5]. Thus, the eddy shedding frequency for the RDSP is modeled as a linear function of the cross flow velocity. For the full scale version, initial sizing estimates are in the range of $100 \mathrm{~m}$ length, $10 \mathrm{~m}$ diameter. Using these sizes as a reference and relative water velocities from 0.1 to $2.0 \mathrm{~m} / \mathrm{s}$, eddies will be shed at frequencies from 0.013 to $0.251 \mathrm{rad} / \mathrm{s}$ (period from 500 to $24 \mathrm{~s}$ ).

Because of the Strouhal number variability and other uncertainties (such as unknown or unmodeled dynamics or sensor resolution/calibration errors), a vortex shedding frequency estimate will likely vary by as much as 15 to $20 \%$ from its actual value. This crude estimate of the frequency provides a strong motivation for using the PLL to better-estimate the disturbance frequency in the loop, as proposed in [7].

\section{Eddy Shedding Force}

The disturbances generated by vortices are nearly periodic and somewhat theoretically predictable [2]. This eddy shedding force is calculated as [21]

$$
F_{\mathrm{L}}(t)=A_{L} \sin \left(\omega_{\mathrm{d}} t\right)
$$

where $A_{L}$ is the lateral eddy shedding force amplitude, $\omega_{\mathrm{d}}$ is the eddy shedding frequency that varies as a function of velocity according to (3), and $t$ is the time in seconds. The amplitude of the force is defined as [1]

$$
A_{\mathrm{L}}=\frac{1}{2} C_{\mathrm{L}} L D \mathrm{\rho} V_{0}^{2}
$$

where $L$ is the length of the section of interest and $C_{\mathrm{L}}$ is the coefficient of lift estimated as 0.28 [21].

\section{CONTROL DESIGN}

Typical control algorithms wait for error accumulate from a disturbance before attempting to compensate for it, but the disturbance rejection technique utilized here has has the unique ability to anticipate and reject disturbances before they induce motions on a system. This pre-existing adaptive control and disturbance rejection algorithm [10] is augmented with a Phase Locked Loop for frequency synthesis. The result of this inclusion is improved performance and robustness with respect to variations in disturbance frequency.

\section{Direct Adaptive Output Regulation And Disturbance Rejection}

Model Reference Adaptive Control (MRAC) with Direct Adaptive Disturbance Rejection (DADR) has been shown to mitigate persistent disturbances of known frequency but unknown amplitude and phase $[10,22-26]$ by taking advantage of periodic or persistent disturbance behavior and adaptively estimating the gains. When applied to large space structures, ocean platforms, wind turbines, and evolving systems $[11,14,27-30]$, this controller has produced good results.

The theoretical development of the controller assumes a Linear Time-Invariant (LTI) plant with the addition of a persistent disturbance, given by

$$
\begin{aligned}
& \dot{x}_{\mathrm{p}}=A_{\mathrm{p}} x_{\mathrm{p}}+B_{\mathrm{p}} u_{\mathrm{p}}+\Gamma_{\mathrm{p}} u_{\mathrm{d}} \\
& y_{\mathrm{p}}=C_{\mathrm{p}} x_{\mathrm{p}} ; \quad x_{\mathrm{p}}(0)=x_{\mathrm{p}}^{0}
\end{aligned}
$$

where $x_{\mathrm{p}}$ is a vector of plant states, $y_{\mathrm{p}}$ is the output, $u_{\mathrm{d}}$ is a persistent disturbance, and $u_{\mathrm{p}}$ is the control command.

When applying the adaptive control algorithm from [10] to a regulation problem, the goal reduces to defining a control law and adaptively adjusting the controller gains such that the plant output, $y_{\mathrm{p}}$, given by (6) will go to zero. This is done in conjunction with adaptive rejection of persistent disturbances. For regulation, the reference system input and state become zero. Thus, the adaptive control law from [10] reduces to

$$
u_{\mathrm{p}}=G y_{\mathrm{p}}+H \phi_{\mathrm{d}}
$$

where $\phi_{\mathrm{d}}$ is a vector that forms a basis for the disturbance. The corresponding adaptive parameter update law given in [10] reduces to

$$
\begin{gathered}
\dot{G}=\Delta_{1} y_{\mathrm{p}} y_{\mathrm{p}}^{\mathrm{T}}-\alpha G, \\
\dot{H}=\Delta_{4} y_{\mathrm{p}} \phi_{\mathrm{d}}^{\mathrm{T}}-\alpha H,
\end{gathered}
$$

where $\Delta_{1}, \Delta_{4}$, and $\alpha$ are design parameters. A block diagram of this control setup is shown in Fig. 3.

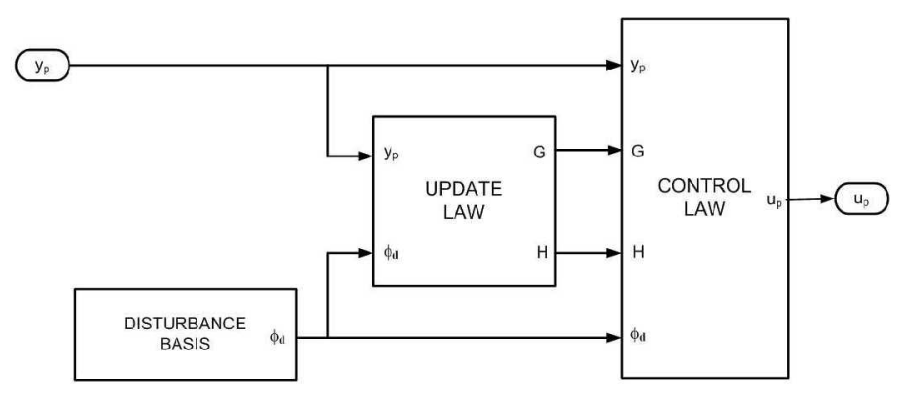

Figure 3. BLOCK DIAGRAM FOR ADAPTIVE OUTPUT REGULATION WITH DIRECT ADAPTIVE DISTURBANCE REJECTION. 


\section{Adaptation For Poorly Known Frequencies}

When the frequency of a disturbance is not precisely known, a complete basis can no longer be predefined for this disturbance. In this case, the performance of the DADR scheme is degraded $[11,14]$. Here, a Phase Locked Loop (PLL) is used to betterestimate the disturbance frequency. This allows the functions in $\phi_{\mathrm{d}}$ within the DADR algorithm to more closely approximate the true basis. The focus of this modification is for the rejection of a single vortex-induced disturbance, but the methodology is also applicable for plants with multiple disturbances.

Phase Locked Loop A Phase Locked Loop (PLL) is a circuit that synchronizes the frequency and phase of an output signal to that of a given input signal $[31,32]$. The PLL that is used here is shown in Fig. 4. Boxes highlight the three main components that are common to all PLLs: synchronized oscillator, phase detector, and loop filter. In general, the role of the

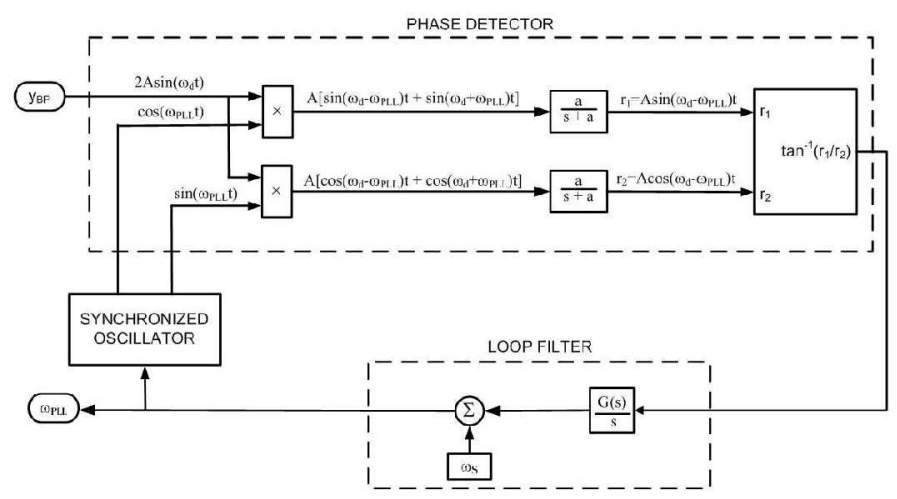

Figure 4. PHASE LOCKED LOOP DETAILS.

three main components may be described as follows. The phase detector component of the PLL compares the input and the Synchronized Oscillator signals to determine an error signal that is proportional to the phase difference between the two. This error feeds through the Loop Filter (LF) and drives the frequency in the opposite direction. By continually updating the frequency in the Synchronized Oscillator in this way, the signal is bettersynchronized with the input signal in terms of phase and frequency. The PLL is designed to reduce the phase error until the output of the LF becomes stationary or "locked", thus inspiring the name "Phase Locked Loop". When operating in this locked state, the frequency exiting the LF will match that of the input signal.

The particular formulation shown in Fig. 4 is a simplified version of [33], and can be found in [34-36] for the case where $G(s)=G$. Through linear analysis of the PLL, $G(s)$ from $[34,35]$ is re-defined as $G(s)=\frac{\left(s+K_{i}\right)}{s}$ to provide an additional zero at the origin of the closed loop transfer function. Its purpose is to cancel with the extra pole generated by a disturbance input with a step change in the frequency rather than in the phase. This modification is made to improve the tracking performance.

Modified Control Framework The control framework set forth in [10] is modified to include an on-line frequency estimate that is provided by a PLL. A block diagram of the adaptive controller and DADR with the inclusion of a PLL is shown in Fig. 5. The RDSP's output goes through a bandpass pre-filter that extracts the component of the signal within the frequency range of interest. This filtered output is used by the PLL to refine the estimate of the disturbance frequency. The PLL frequency estimate is lowpass filtered to eliminate any high frequency oscillations. Finally, this frequency replaces the static frequency guess in the DADR basis functions.

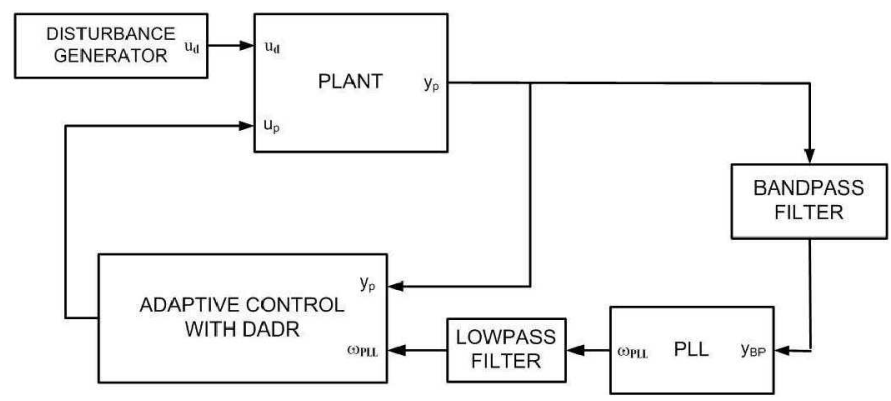

Figure 5. CLOSED LOOP SYSTEM WITH MRAC/DADR AND A PLL.
The bandpass pre-filter allows all of the signals outside the range of interest to be removed. Therefore, the input to the PLL primarily contains the component of the plant's output that is a direct result of the disturbance. If multiple disturbances are expected, the framework may be adapted by including additional sets of PLLs with corresponding filters. For example, $n$ disturbance frequency estimates would require $n$ sets of bandpass filters, PLLs, and low pass filters. Each bandpass pre-filter will be adjusted according to the expected frequency of the corresponding disturbance.

The filtered PLL frequency, $\omega_{P L L}^{L P}$ is used in the DADR scheme rather than the unfiltered frequency, $\omega_{\mathrm{PLL}}$, to reduce the oscillations in the DADR basis functions. This also reduces the effect of the disturbance rejection portion of the controller at the beginning of the simulation, giving the PLL time to lock onto the desired frequency without having a large amount of interaction with the DADR. 


\section{RESULTS}

For each 6-DOF simulation, the six forward facing propellers provide a constant thrust of $300 \mathrm{~N}(50 \mathrm{~N}$ each) in the $x$-direction, inducing a surge velocity of approximately $0.22 \mathrm{~m} / \mathrm{s}$. An initial surge velocity of $0.22 \mathrm{~m} / \mathrm{s}$ is given to the RDSP but there is still a transient period before the disturbance frequency converges to a constant value (Fig. 7, Fig. 11). A basic PID controller is used to minimize the RDSP's yaw motion while the adaptive controller is configured to reduce the transverse oscillatory motions. As with the 1 DOF model $[7,14]$, the control law given by (7) with parameter update laws given by (8) is used in conjunction with a PLL. This controller implementation is shown in Fig. 5.

The gains in the update law (8) are chosen to be $\Delta_{1}=$ $200,000 I_{2}, \Delta_{4}=20,000 I_{2}$, and $\alpha=1 \cdot 10^{-4}$. For the PLL, the gains are chosen to yield negative (stable) roots for $s^{3}+a s^{2}+$ $a G s+a G K_{i}=0$ by selecting $G=2.5 \cdot 10^{-4}, K_{i}=2.844 \cdot 10^{-6}$, and $a=0.3$.

The frequency guess for vortex disturbances was taken as $\omega_{S}=0.25 \mathrm{rad} / \mathrm{s}$. This is approximately $78 \%$ of the steady-state frequency of vortex-induced disturbances $\left(\omega_{\mathrm{d}} \approx 0.32\right)$. Thus, $\left|\omega_{d}-\omega_{s}\right|$ is selected such that it exceeds the expected frequency estimation error for this application.

For this simulation, two sensor/actuator configurations are considered, as shown in Fig. 6. The first is a Single-Input-Single

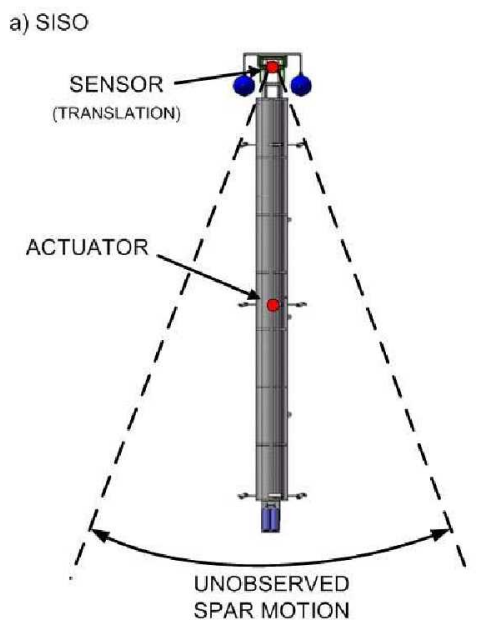

a) MIMO

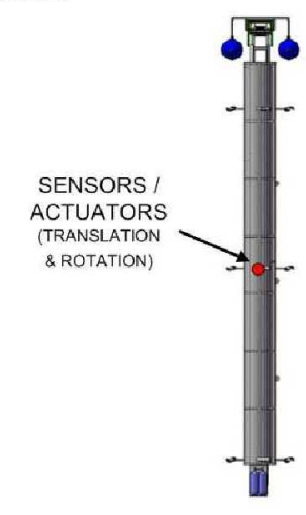

Figure 6. SENSOR AND ACTUATOR LOCATIONS.

Output (SISO) scenario, where the sensor is located at a point on the catamaran (near the top of the spar) to detect motion in the $y$-direction. The control input is taken to be a point force acting at the location of the central thruster, or equivalently equal thrust from all lateral thrusters. For the second simulated configuration, the input and output are collocated at the center thrusters. In this case, the output contains both the sway and roll velocities and the input is the force and moment in these directions. These outputs can be estimated by measuring the motions at the catamaran, where the sensor system is currently located on the RDSP prototype, and transforming these measurements to motion estimates at the center set of thrusters. The force input can be applied to the physical system by dividing the total desired force equally among each of the lateral thrusters, as with the SISO simulation. The moment portion of the input can be achieved by superimposing equal and opposite thrusts from the top and bottom sets of lateral thrusters.

The effectiveness of the controller when used in conjunction with an on-line frequency estimate was shown for a simple 1DOF simulation in $[7,14]$ and will now be verified for a 6-DOF RDSP model with increased dynamical complexity.

\section{Single-Input-Single-Output Scenario}

For the SISO scenario, the output is taken to be the velocity of the $1 / 10^{\text {th }}$ scaled prototype in the $y$-direction at the top of the spar. The input is the force in the $y$-direction, applied at the middle thrusters (or equally across each of the lateral thrusters). This simple sensor/actuator configuration may be able to realize the goal of mitigating the vortex-induced transverse oscillatory motion at the top of the spar, but the control system is not capable of intentionally eliminating RDSP pendulations about this point since they are not visible in the output (see Fig. 6).

The on-line frequency estimate is shown in Fig. 7. In Fig.

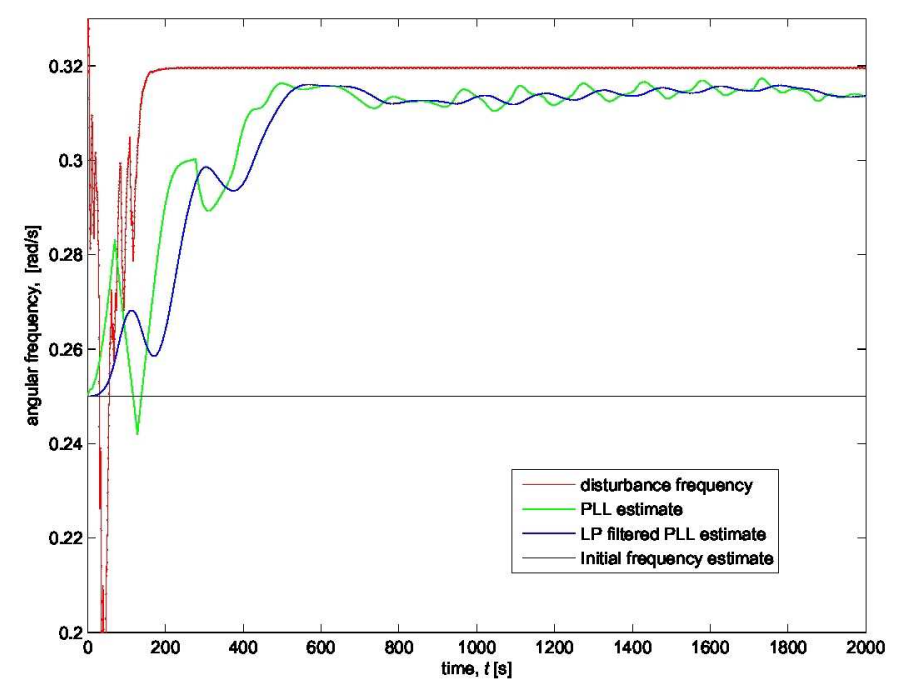

Figure 7. DISTURBANCE FREQUENCY ESTIMATE FOR THE SISO SIMULATION.

8 , the disturbance is shown alongside the DADR portion of the 
controller at the output (sensor) location. The standard devia-
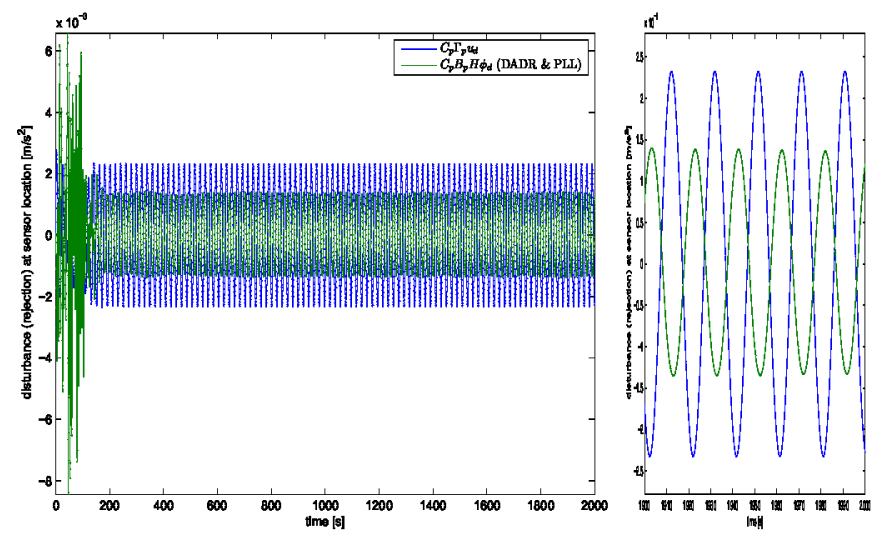

Figure 8. DISTURBANCE REJECTION EFFECTIVENESS FOR THE SISO SIMULATION: FULL RUN TIME (LEFT) AND LAST $100 \mathrm{~s}$ (RIGHT).

tion of the acceleration at this point due to the vortex-induced disturbance, given by $C_{\mathrm{p}} \Gamma_{\mathrm{p}} u_{\mathrm{d}}$ is $1.623 \cdot 10^{-3} \mathrm{~m} / \mathrm{s}^{2}$. When the DADR with a PLL is included, the STD of $\left(C_{\mathrm{p}} \Gamma_{\mathrm{p}} u_{\mathrm{d}}+C_{\mathrm{p}} B_{\mathrm{p}} H \phi_{\mathrm{d}}\right)$ becomes $1.056 \cdot 10^{-3} \mathrm{~m} / \mathrm{s}^{2}$, and thus reduces the disturbance appearing in the output by $35 \%$.

The portion of the error not rejected a priori by the DADR scheme enters into the dynamical system (RDSP) and must then be mitigated by the output feedback term in the adaptive control law. The open loop STD of the velocity resulting from the vortex shedding for the last $1000 \mathrm{~s}$ of the simulation is $3.046 \cdot 10^{-3} \mathrm{~m} / \mathrm{s}$. If the loop is closed with the MRAC (MRAC/DADR), this is reduced to $2.626 \cdot 10^{-3} \mathrm{~m} / \mathrm{s}\left(1.300 \cdot 10^{-4} \mathrm{~m} / \mathrm{s}\right)$. The inclusion of the PLL with the MRAC/DADR algorithm yields a transverse velocity error with a STD of $6.4 \cdot 10^{-5}$. Thus, the controller nearly eliminates the velocity error, $\dot{y}_{\mathrm{p}}$, at the top of the spar (as shown in Fig. 9). After removing the bias, the open and closed loop displacements are shown in Fig. 10 for the latter $1000 \mathrm{~s}$ of the simulation. As with the velocity, the oscillations in position are almost completely eliminated.

\section{Multi-Input-Multi-Output Scenario}

The output for the MIMO example is a vector containing the sway velocity in $\mathrm{m} / \mathrm{s}$ and 10 times the roll velocity in $\mathrm{rad} / \mathrm{s}$. The sway velocity output signal is selected as the input to the PLL, which provides an estimate for the frequency of both the force and torque disturbances. Because the disturbance frequency itself oscillates about an equilibrium, the PLL gains were selected to provide a somewhat sluggish response. Thus, it takes $600 \mathrm{~s}$ for the frequency estimate to initially approach the actual disturbance frequency (as with the SISO case). This is shown in Fig. 11.

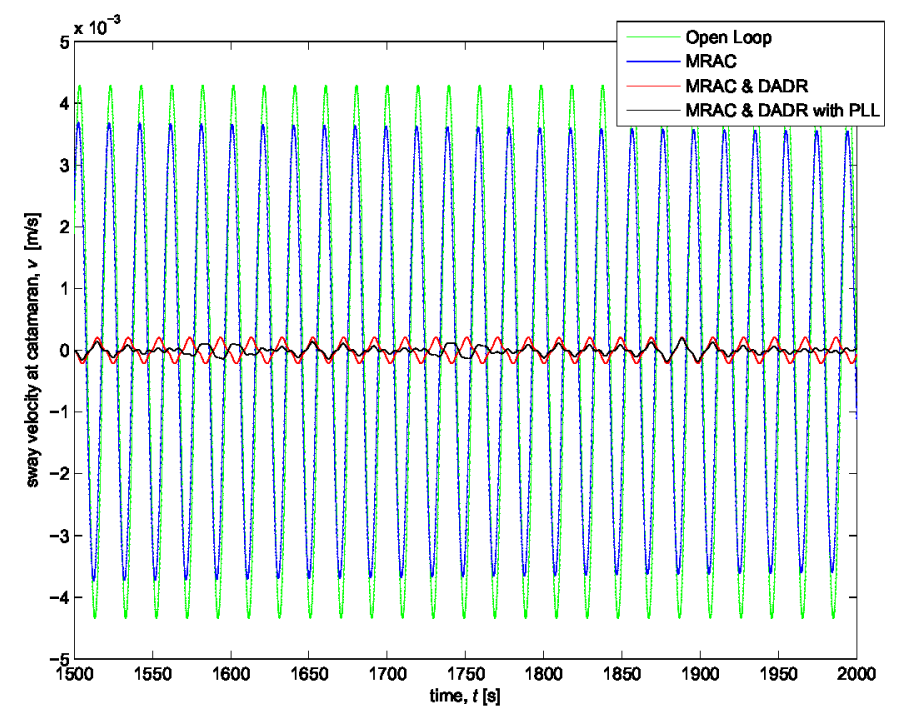

Figure 9. SWAY VELOCITY COMPARISON FOR THE SISO SIMULATION.

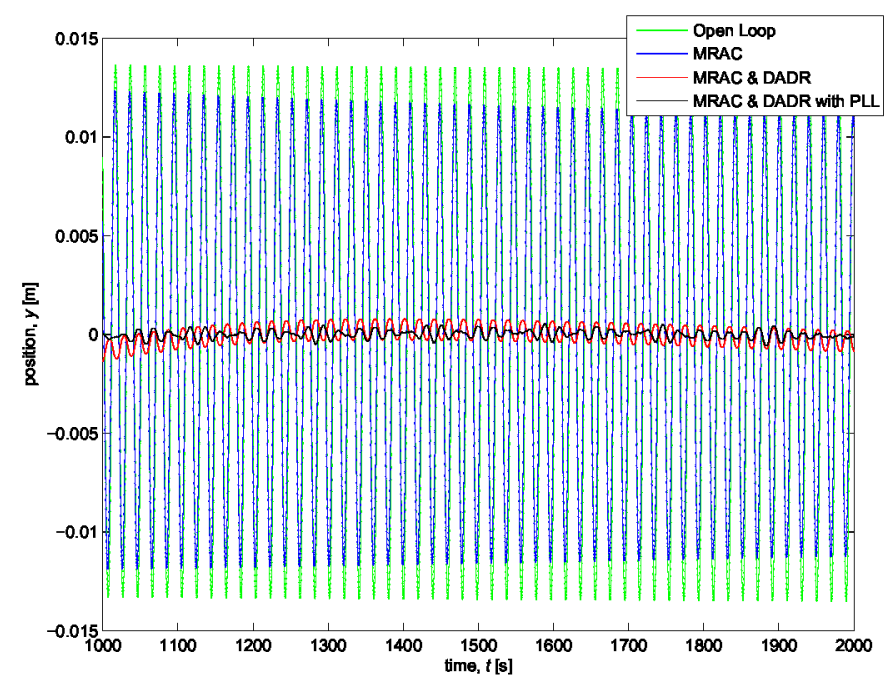

Figure 10. TRANSVERSE DISPLACEMENT COMPARISON FOR THE SISO SIMULATION.

Fig. 12 shows the disturbance input $\left(u_{\mathrm{d}}\right)$ plotted against the DADR component of the control command $\left(H \phi_{d}\right)$ with and without the inclusion of a PLL. This is shown over only a portion of the simulation time in order to provide a closer look at these signals. The standard deviation of the disturbance force and moment over the last $1000 \mathrm{~s}$ of the simulation are $49.82 \mathrm{~N}$ and $36.05 \mathrm{~N}-\mathrm{m}$, respectively. The disturbance rejection component of the control command, $H \phi_{\mathrm{d}}$, does a much better job at opposing the disturbance signal, $u_{\mathrm{d}}$, than in the SISO scenario. This may also be observed statistically by again considering the unre- 


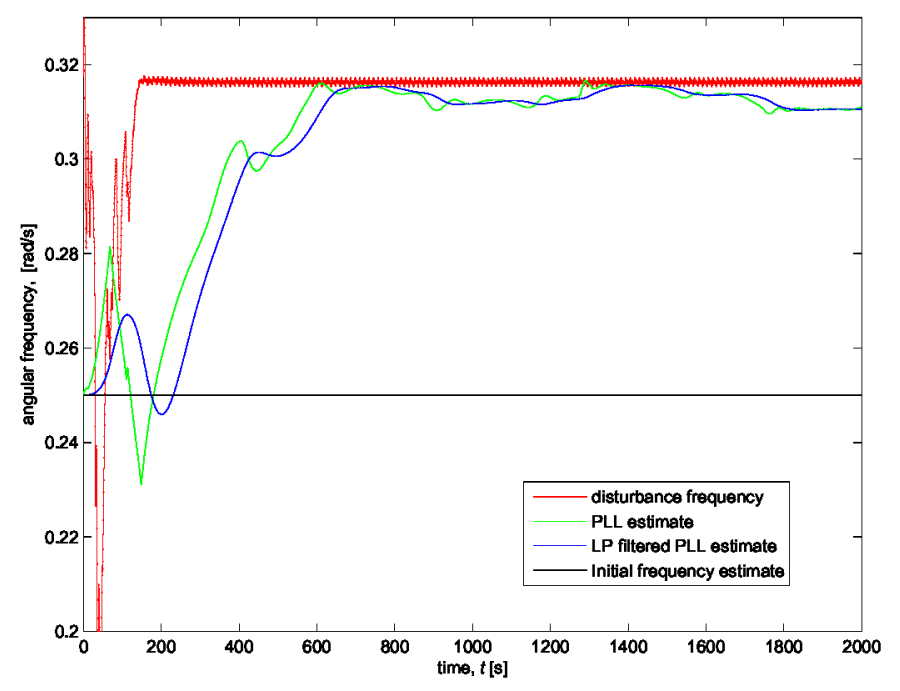

Figure 11. DISTURBANCE FREQUENCY ESTIMATE FOR THE MIMO SIMULATION.
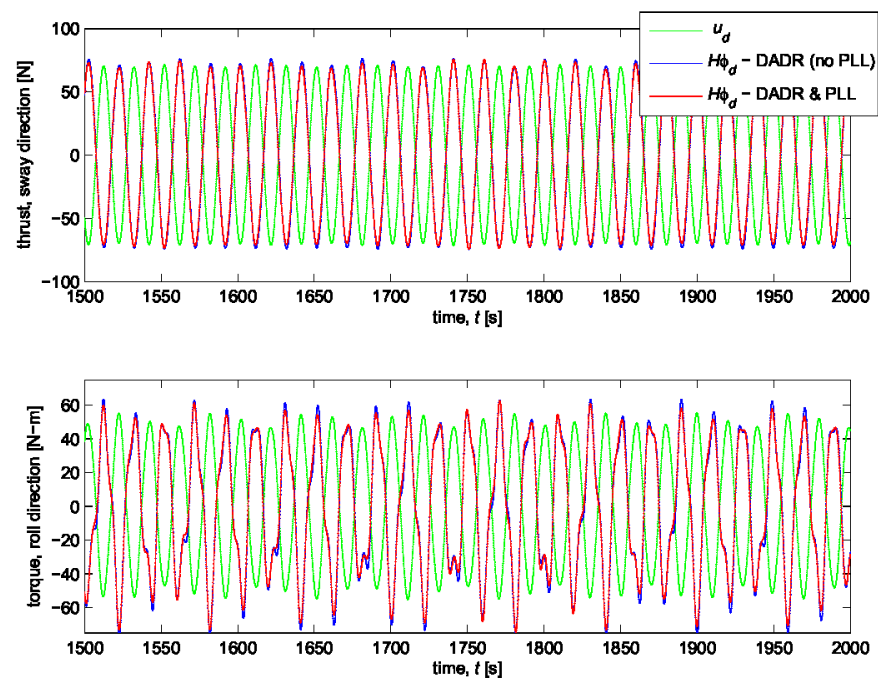

Figure 12. EFFECTIVENESS OF DISTURBANCE REJECTION FOR THE MIMO SIMULATION.

jected portion of the disturbance. Without the inclusion of a PLL, $\operatorname{STD}\left(u_{\mathrm{d}}+H \phi_{\mathrm{d}}\right)$ is $2.55 \mathrm{~N}$ (95\% reduction) for the sway disturbance component and $10.54 \mathrm{~N}-\mathrm{m}$ ( $71 \%$ reduction) for the roll disturbance component. When these STD values are calculated again with the inclusion of a PLL, the result is $1.47 \mathrm{~N}$ (97\% reduction) for the sway disturbance and $8.55 \mathrm{~N}-\mathrm{m}$ ( $76 \%$ reduction) for the roll disturbance. Thus, approximately three times as much of the disturbance is rejected a priori for this sensor/actuator scenario (and, in fact, almost all of it).

As could be expected based on the effectiveness of the DADR component alone, the controller results in a significant reduction in output error (sway and roll velocities) in all cases. For the open loop results, the sway velocity has a standard deviation of $7.1 \cdot 10^{-3} \mathrm{~m} / \mathrm{s}$ and the standard deviation of the roll velocity is $3.88 \cdot 10^{-2} \mathrm{deg} / \mathrm{s}$ at the center thruster over the last $1000 \mathrm{~s}$. When the MRAC/DADR with PLL is applied, the STD is reduced to $1.31 \cdot 10^{-4} \mathrm{~m} / \mathrm{s}$ for the sway velocity and $8.36 \cdot 10^{-3}$ $\mathrm{deg} / \mathrm{s}$ for the roll velocity. This corresponds to a reduction in error of $98.2 \%$ for sway velocity, $v$ - more than a two-fold increase in overall output error reduction when compared to the SISO results. The roll velocity, $p$, was similarly reduced by $95.2 \%$ (this DOF was not controlled in the SISO case). The corresponding displacements in the sway direction are shown in Fig. 13, and the roll angles are shown in Fig. 14.

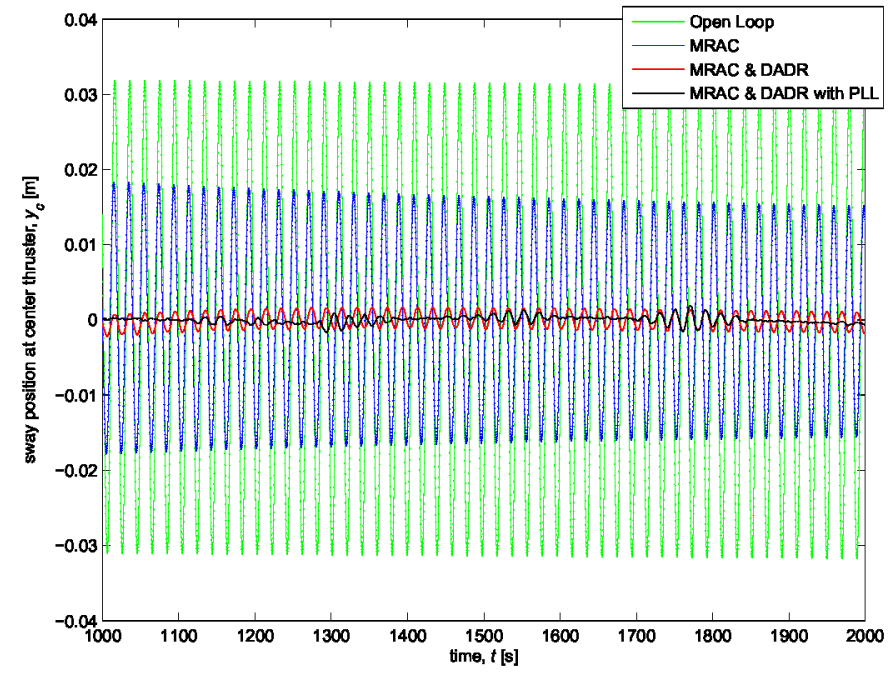

Figure 13. TRANSVERSE DISPLACEMENT COMPARISON FOR THE MIMO SIMULATION.

\section{CONCLUSIONS}

An adaptive control and disturbance rejection algorithm with the inclusion of disturbance frequency synthesis was shown to mitigate vortex-induced motions for the RDSP operating as a spar platform in VOM. Simulation results were shown for two 6DOF RDSP actuator/sensor scenarios: (i) a single control force located at center thruster and a single output at the top of the spar, and (ii) control actuation using both force and moment generated at the center thruster with two collocated outputs. The location of the actuators and sensors in the latter (MIMO) scenario reduced the transverse oscillitory motion to half of that found for the former (SISO) scenario. In both cases, the inclusion of a PLL allowed the MRAC/DADR to more effectively mitigate the persistent, vortex-induced disturbance at a frequency that was not well known. 


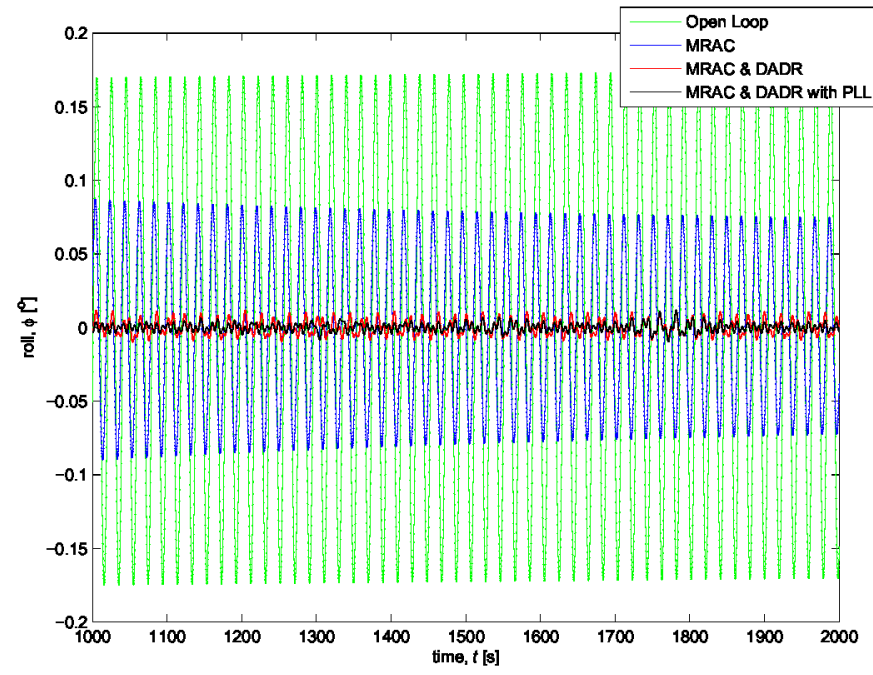

Figure 14. ROLL ANGLE COMPARISON FOR THE MIMO SIMULATION.

\section{ACKNOWLEDGMENT}

The authors gratefully acknowledge the NASA Graduate Student Researchers Program and the Marshall Space Flight Center for partial support of this research.

The authors also acknowledge the Office of Naval Research, code 33, program manager Kelly Cooper for partially funding this work under grant N00014-06-1-0461.

\section{REFERENCES}

[1] Crowe, C. T., Robertson, J. A., and Elger, D. F., 2001. Engineering Fluid Mechanics, 7th ed. John Wiley \& Sons, Inc., New York, NY.

[2] Fischer, F. J., Liapis, S., and Lallinderis, Y., 2004. "Mitigation of current-driven vortex-induced vibrations of a spar platform via "smart" thrusters". Journal of Offshore Mechanics and Arctic Engineering, 126, February, pp. 96-104.

[3] Schulz, K., and Kallinderis, Y., 2000. "Numerical predictions of the hydrodynamic loads and vortex-induced vibrations of offshore structures". ASME Journal of Offshore Mechanics and Arctic Engineering, 122, pp. 289-293.

[4] Allen, D. W., and Henning, D. L., 1997. "Vortex-induced vibration tests of a flexible smooth cylinder at supercritical reynolds numbers". In Proceedings of International Offshore and Polar Engineering Conference, pp. 680-685.

[5] Skop, R. A., and Luo, G., 2001. "An inverse-direct method for predicting the vortex-induced vibrations of cylinders in uniform and nonuniform flows". Journal of Fluids and Structures, 15, February, pp. 867-884.

[6] VanZwieten, J., Driscoll, F., and Alsenas, G., 2008. "Response characteristics and maneuverability of a small twin screw displacement hull vessel in seas". Ships and Offshore Structures, 3(1), pp. 13-40.

[7] VanZwieten, J. H., VanZwieten, T. S., Balas, M. J., and Driscoll, F. R., 2009. "Mitigation of vortex-induced disturbances for the rapidly deployable stable platform". Journal of Ships and Offshore Structures, January. Submitted.

[8] Marikle, S., Ravenna, S., VanZwieten, J., Alsenas, G., and Driscoll, F. Development of a $1 / 10^{\text {th }}$ scale rapidly deployable stable platform. Preprint, 2008.

[9] VanZwieten, J., Driscoll, F., and VanZwieten, T. Development of an adaptive disturbance rejection system for the rapidly deployable stable platform - Part 1: Mathematical modeling and open loop response. preprint, 2008.

[10] Fuentes, R. J., and Balas, M. J., 2002. "Robust model reference adaptive control with disturbance rejection". In Proceedings of American Control Conference, Vol. 5, pp. 4003-4008.

[11] VanZwieten, T. S., VanZwieten, J. H., Driscoll, F. R., and Balas, M. J., 2008. "Adaptive disturbance rejection for the rapidly deployable stable platform when transferring cargo in seas". In Proceedings of MTS/IEEE OCEANS Conference.

[12] VanZwieten, T. S., Balas, M. J., and VanZwieten, J. H., 2008. "Adaptive output tracking and disturbance rejection with saturation constraints". In Proceedings of AIAA Guidance Navigation \& Control Conference.

[13] VanZwieten, T. S., VanZwieten, J. H., Balas, M. J., and Driscoll, F. R. Development of an adaptive disturbance rejection system for the rapidly deployable stable platform - Part 2: Controller design and closed loop response. preprint, 2008.

[14] VanZwieten, T. S., 2008. "Adaptive control for applications with input saturation constraints or disturbances at poorly known frequencies". PhD thesis, University of Wyoming, Laramie, WY.

[15] Driscoll, F. R., Venezia, A., Alsenas, G., Galvan, F., Radanovic, B., and Selfridge, M., 2006. "A scale model rapidly deployable stable craneship for seabasing cargo transfer". In Proceedings of the $16^{\text {th }}$ International Offshore and Polar Engineering Conference and Exhibition, no. 2006-jsc-286.

[16] Newman, J. N., 1977. Marine Hydrodynamics. The MIT Press, Cambridge, Massachusetts and London, England.

[17] Lewis, E. V., 1988. Principles of Naval ArchitectureVolume II: Resistance, Propulsion, and Vibration, Vol. 2. The Society of Naval Architects and Marine Engineers, Jersey City, New Jersey.

[18] Deep Oil Technologies, Inc, 1995. Spar model test joint industry project: Final report. Tech. Rep. R930501, September.

[19] Selfridge, M. J., 2005. "Spar technology as a seabasing enabler". In Proceedings of ASNE Seabasing Conference. 
[20] Thrustmaster of TeXas, 2001. General Technical Data Sheet for Thrustmaster Model TH-300N Thru-Hull Azimuthing Thruster. Houston, TX.

[21] Skop, R. A., and Balasubramanian, S., 1997. "A new twist on an old model for vortex-excited vibrations". Journal of Fluids and Structures, 11, pp. 395-412.

[22] Wen, J., and Balas, M. J., 1989. "Robust adaptive control in hilbert space". Journal of Mathematical Analysis and Application, 143, pp. 1-26.

[23] Balas, M., 1995. "Finite-dimensional direct adaptive control for discrete-time infinite-dimensional linear systems". Journal of Mathematical Analysis and Applications, 196, pp. 153-171.

[24] Fuentes, R. J., and Balas, M. J., 2000. "Direct adaptive rejection of persistent disturbances". Journal of Mathematical Analysis and Applications, 251(1), November, pp. 2839.

[25] Balas, M., Robertson, L., and Fuentes, R., 2006. "Tracking and disturbance rejection of inaccessible signals in linear systems". In Proceedings of American Control Conference, pp. 5655-5660.

[26] Balas, M. J., Harvey, S., and Mehiel, E., 2008. "Nonlinear adaptive control with persistent disturbances". In Proceedings of AIAA Guidance Navigation \& Control Conference.

[27] Balas, M. J., Erwin, R. S., and Fuentes, R., 2000. "Adaptive control of persistent disturbances for aerospace structures". In Proceedings of AIAA Guidance Navigation \& Control Conference.

[28] Mehiel, E., Balas, M., and Fuentes, R., 2002. "A discrete time direct adaptive disturbance rejection and control algorithm applied to a model of a deployable space telescope". In Proceedings of AIAA Guidance Navigation \& Control Conference.

[29] Fuentes, R., Schrader, K., Balas, M., and Erwin, R. S., 2001. "Direct adaptive disturbance rejection and control for a deployable space telescope, theory and application". In Proceedings of American Control Conference.

[30] VanZwieten, T. S., and Balas, M. J., 2007. "Evolving systems: Inheriting stability with decentralized adaptive control". In Proceedings of AIAA Guidance Navigation \& Control Conference.

[31] Best, R. E., 2007. Phase-Locked Loops: Design, Simulation, and Applications, 6 ed. The McGraw-Hill Companies, Inc., New York, NY.

[32] Hsieh, G.-C., and Hung, J. C., 1996. "Phase-locked loop techniques - a survey". IEEE Transactions on Industrial Electronics, 43, December, pp. 609-615.

[33] Chiang, W.-W., and Robert H. Cannon, J., 1985. "The experimental results of a self tuning adaptive controller using online frequency identification". Journal of Astronautical Sciences, 33, Jan-Mar, pp. 71-83.

[34] Ouyang, J. J., and Balas, M. J., 1986. "Self tuning adaptive controller using on line frequency locked loops". In Proceedings of Asilomar Conference on Circuits, Systems and Computers.

[35] Ouyang, J., 1987. "Adaptive residual mode filter control of dps for large space structure application". PhD thesis, Rensseler Polytechnic Institute, Troy NY, May.

[36] Davidson, R. A., Balas, M. J., and Reisenauer, B. T., 1990. "Adaptive csi compensation for reduced-ordermodel-based control of a flexible robot manipulator". In Proceedings of American Control Conference, pp. 334 339. 